\title{
Effect of ECG Filtering on Time Domain Analysis of the P-Wave
}

\author{
F Censi ${ }^{1}$, G Calcagnini ${ }^{1}$, P Bartolini $^{1}$, E Cervi $^{2}$, \\ I Diemberger $^{2}$, I Corazza $^{2}$, G Boriani $^{2}$ \\ ${ }^{1}$ Department of Technologies and Health, Italian Institute of Health, Rome, Italy \\ ${ }^{2}$ Institute of Cardiology, University of Bologna, Policlinico S Orsola, 40138 Bologna, Italy
}

\begin{abstract}
The aim of this study was to assess the effect of filtering techniques on the time-domain analysis of the Pwave. Multi-lead ECG recordings obtained from chronic AF patients after successful external cardioversion have been acquired. Several high-pass filtering techniques and three cut-off frequency values were used: Bessel and Butterworth four-pole and two-pole bidirectional and unidirectional filters, at 0.01, 0.05 and $0.5 \mathrm{~Hz}$ low cut-off frequency. As a reference, a beat-by-beat linear piecewise interpolation was used to remove baseline wander, on each P-wave. Results show that ECG filtering affects the estimation of $P$-wave duration in a manner that depends upon the type of filter used: particularly, the bidirectional filters caused negligible variation of $P$ wave duration, while unidirectional ones provoked an increase higher than $8 \%$.
\end{abstract}

\section{Introduction}

Atrial fibrillation (AF) [1][2] is the major cardiac rhythm disturbance associated with significant morbidity and mortality [3]. Normal cardiac impulse initiation begins in the sinoatrial node and then travels through working atrial myocardium.

AF is associated to the spread of atrial depolarization pattern across the atria in a manner different from the normal through accessory pathways, reentries or conduction abnormalities.

Abnormal P-waves have been observed in patients prone to $\mathrm{AF}$ and the analysis of $\mathrm{P}$-wave from surface electrocardiogram has been extensively used to identify patients prone to atrial arrhythmias, especially AF [12][4-8].

In this context, P-wave analysis is usually performed following both the conventional 12-leads ECG approach and the three bipolar orthogonal leads one, used to construct a P-wave vector magnitude. Given the relatively low $\mathrm{P}$-wave amplitude respect to background noise, both approaches use signal averaging technique to obtain a P-wave template.

The quantitative analysis of the P-wave template turned out to be a discriminative test for patients at risk of developing Atrial Fibrillation (AF) or with paroxymal AF with high sensitivity and specificity. The most reproducible parameter seems to be the $\mathrm{P}$-wave duration.

Indeed, slowed conduction velocity in several atrial regions together with different cell refractory periods, are believed to be the electrophysiological conditions provoking and maintaining AF [9].

Such atrial conduction abnormalities result in prolonged and highly variable P-waves, detectable by surface ECG.

However, the estimation of P-wave duration, performed either manually or automatically, can be affected by the filtering of the ECG signal applied by the acquisition system used.

These systems differ mainly in the type of filters and filtering frequencies used before the signal processing and the extraction of quantitative variables.

The main purpose of the high-pass filter in electrocardiograph equipment is to suppress baseline wander.

Review of high-pass filter settings on various modern electrocardiographs shows that manufacturers provide a number of different options., in terms of cut-off frequency, filter order and filter type.

The aim of this study was to assess the effect of filtering techniques on the time-domain analysis of the Pwave.

\section{Methods}

Multi-lead ECG obtained from 10 chronic AF patients after successful external cardioversion have been acquired using a mapping system for high-resolution biopotential measurement (ActiveTwo, Biosemi, The Netherlands), sample frequency $2 \mathrm{kHz}, 24$ bit resolution, 0-400 Hz bandwidth.

The system is made of a battery powered AD box that digitises the signals and transfers them to a PCI receiver 
on computer through fiber optic connection.

Particularly, the 16-lead configuration showed in figure 1 has been used. ECG recording lasted 5-minute, with the patient laying on the bed in resting position.

The experimental protocol foresees a first ECG recording soon after the external cardioversion and successive ECG recordings after 6 and 12 months.

At this stage of the project, all patients have been undergone only to the first ECG recording.

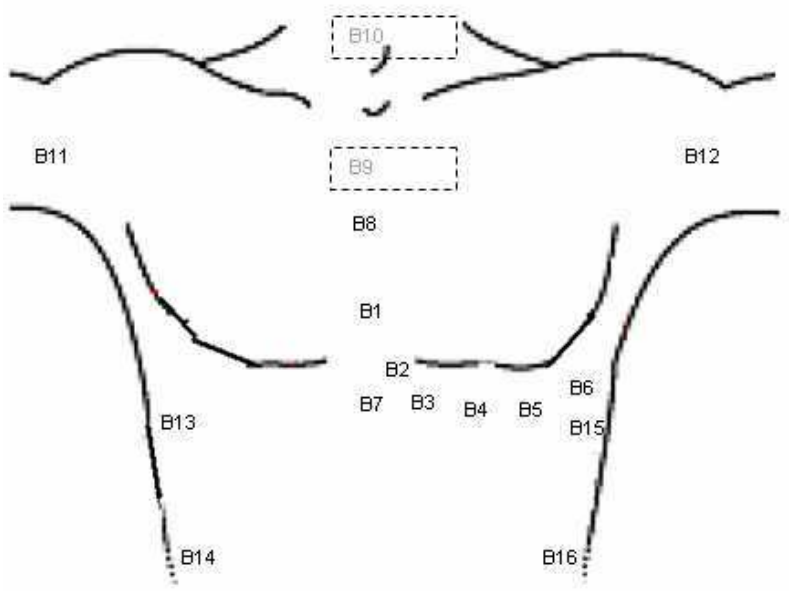

Figure 1. Electrodes position used for the multi-lead ECG acquisition. Electrodes are indicated with the letter B prefix. Electrode B9 and B10 (light gray in dashed line box) are positioned on the back.

\subsection{High-pass filtering}

To simulate the analog filters implemented in the most widely used ECG commercial devices, we designed digital high-pass filters, which we applied to the raw ECG recording obtained by the DC-coupled ECG acquisition system used in this study.

Bessel and Butterworth four-pole and two-pole bidirectional and unidirectional filters were used at the tree cut-off frequency values of $0.01,0.05$ and $0.5 \mathrm{~Hz}$, summarized in table 1.

Bidirectional filter was implemented by filtering the signal in the forward direction and then filtering again in the reverse direction. In this way the magnitude of the frequency response is the same for each direction, while the phases are opposite in sign. When the two directions are combined, the magnitude becomes squared, while the phase cancels to zero.

As a reference, a beat-by-beat linear piecewise interpolation was used to remove baseline wander, on each P-wave. Fiducial points for linear interpolation were taken from TP and PQ tracks of each beat.

After ECG filtering, every lead signal was pre- processed and analysed to extract the average $\mathrm{P}$-wave ( $\mathrm{P}$ wave template): after $\mathrm{P}$-wave detection, a coherent averaging procedure is applied by aligning the $\mathrm{P}$-waves according to the lag at which the cross-correlation function between the current averaged P-wave and each single P-wave shows its maximum. The coherent averaging procedure went on until 200 beats were included. If the residual noise level remained at more than $1 \mu \mathrm{V}$ even after averaging of 200 beats, averaging procedure continued until the noise level reached a value lower than $1 \mu \mathrm{V}$. If it was impossible, the lead was excluded from the study. Residual noise was measured in the isoelectric segment before the P-wave (TP track) [10].

Table 1. Index of the filters tested. Uni=unidirectional; $\mathrm{Bi}=$ bidirectional.

\begin{tabular}{|l|l|l|l|l|}
\hline Filter & type & $\begin{array}{l}\text { Uni/bi } \\
\text { directional }\end{array}$ & $\begin{array}{l}\text { Cut-off } \\
\text { frequency } \\
\text { (Hz) }\end{array}$ & order \\
\hline BeU01_2 & Bessel & Uni & 0.01 & 2 \\
\hline BeU01_4 & Bessel & Uni & 0.01 & 4 \\
\hline BeU05_2 & Bessel & Uni & 0.05 & 2 \\
\hline BeU05_4 & Bessel & Uni & 0.05 & 4 \\
\hline BeU5_2 & Bessel & Uni & 0.5 & 2 \\
\hline BeU5_4 & Bessel & Uni & 0.5 & 4 \\
\hline BeB01_2 & Bessel & $\mathrm{Bi}$ & 0.01 & 2 \\
\hline BeB01_4 & Bessel & $\mathrm{Bi}$ & 0.01 & 4 \\
\hline BeB05_2 & Bessel & $\mathrm{Bi}$ & 0.05 & 2 \\
\hline BeB05_4 & Bessel & $\mathrm{Bi}$ & 0.05 & 4 \\
\hline BeB5_2 & Bessel & $\mathrm{Bi}$ & 0.5 & 2 \\
\hline BeB544 & Bessel & $\mathrm{Bi}$ & 0.5 & 4 \\
\hline BuU01_2 & Butterworth & Uni & 0.01 & 2 \\
\hline BuU01_4 & Butterworth & Uni & 0.01 & 4 \\
\hline BuU05_2 & Butterworth & Uni & 0.05 & 2 \\
\hline BuU05_4 & Butterworth & Uni & 0.05 & 4 \\
\hline BuU5_2 & Butterworth & Uni & 0.5 & 2 \\
\hline BuU5_4 & Butterworth & Uni & 0.5 & 4 \\
\hline BuB01_2 & Butterworth & Bi & 0.01 & 2 \\
\hline BuB01_4 & Butterworth & $\mathrm{Bi}$ & 0.01 & 4 \\
\hline BuB05_2 & Butterworth & $\mathrm{Bi}$ & 0.05 & 2 \\
\hline BuB05_4 & Butterworth & $\mathrm{Bi}$ & 0.05 & 4 \\
\hline BuB5_2 & Butterworth & $\mathrm{Bi}$ & 0.5 & 2 \\
\hline BuB5_4 & Butterworth & $\mathrm{Bi}$ & 0.5 & 4 \\
\hline
\end{tabular}

\subsection{P-wave duration}

P-wave duration has been automatically calculated by using the algorithm described in [12]. Briefly, P-wave duration is defined as the time between the onset and the offset of the P-wave. P-wave onset is automatically computed as the first point, among 20 consecutive points, 
higher than 3 times the residual noise standard deviation. Offset is analogously defined considering the first point, among the last 20 consecutive samples going backward from the QRS, being above the same threshold. Residual noise was measured in the isoelectric segment before the $\mathrm{P}$-wave, as for the coherent averaging procedure stop criteria.

\section{Results}

Results show that ECG filtering affects the estimation of P-wave duration. Such effects occur in a manner that depends upon the type of filter used.

Particularly, the cut-off frequencies tested in this study, seems to affect the P-wave duration in similar manner. As for the filter order, we found that the 2-pole filters provoke the P-wave duration to be 1-2 ms longer than the 4-pole ones, and thus affect the P-wave duration estimation in a negligible manner.

Respect to the not-filtered $\mathrm{P}$-wave, the bidirectional filters caused a not significant variation of $\mathrm{P}$-wave duration, while unidirectional ones provoked longer Pwaves. The average increase of $\mathrm{P}$-wave duration for the BuU5_4 filter was $8.7 \% \pm 6.1 \%$. Similar results have been obtained for the other filters. An example of original and filtered ECG is showed in figure 2. In this case P-wave duration estimated from the P-wave template extracted from the original unfiltered signal was equal to $127 \mathrm{~ms}$, while $\mathrm{P}$-wave duration values estimated from the $\mathrm{P}$-wave template extracted from the $\mathrm{BuB5} 4$ and from the BuU5_4 filtered ECG recordings were $131 \mathrm{~ms}$ and 135 $\mathrm{ms}$, respectively.

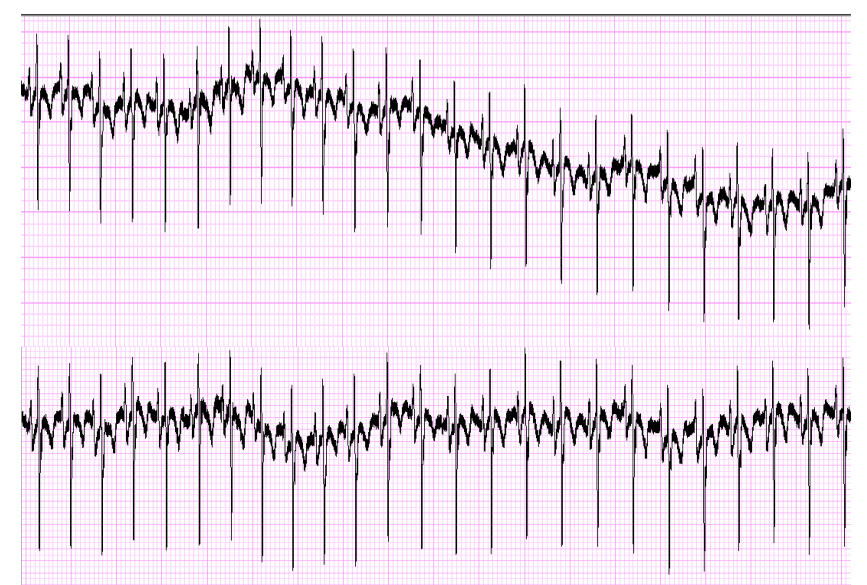

Figure 2. Example of original (upper panel) and highpass filtered ECG.

Figure 3 shows an example of P-wave templates extracted from an original (upper panel) and an ECG filtered using a Butterworth unidirectional 4-pole filter at $0.5 \mathrm{~Hz}$ (lower panel). Note the different morphologies of the $\mathrm{P}$-waves and the consequent variation in the $\mathrm{P}$-wave duration estimation.

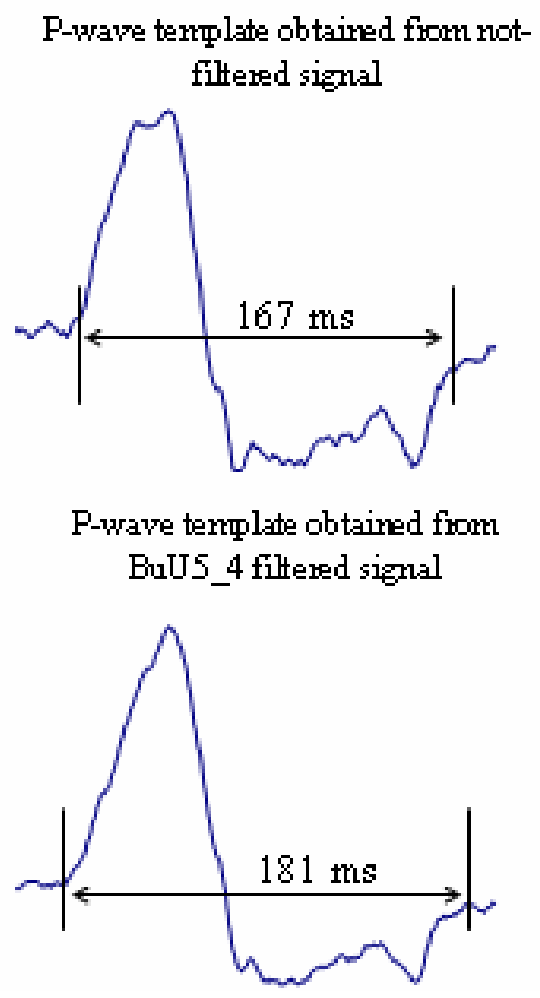

Figure 3. Example of P-wave templates extracted from an original (upper panel) and a filtered (lower panel) ECG.

\section{Discussion and conclusions}

The quantitative analysis of the P-wave is widely used to discriminate patients at risk of developing AF. Atrial conduction abnormalities and atrial impulse propagation delays provoke the P-wave to be prolonged and morphologically complicated after restoring to sinus rhythm, respect to the P-wave of a patient who never experienced AF.

However the quantitative analysis of the $\mathrm{P}$-wave is often performed without taking into account important technical aspects of the ECG acquisition and processing: different acquisition settings and processing algorithms used in different clinical realities cause the results to be incomparable.

In addition, many techniques used to extract quantitative indexes concerning the $\mathrm{P}$-wave prolongation 
or complexity are still based on manual indication by visual inspection.

Particularly, for the estimation of the P-wave duration, various filtering techniques and algorithms are used for the detection of the onset and offset of the P-wave.

Given the low-amplitude of the P-wave portion of the ECG, the detection of such fiducial points is crucial. Thus it becomes necessary to investigate the effect of ECG acquisition and pre-processing procedure on the detection and estimation of the P-wave characteristics.

This issue has been investigated for the signalaveraged ECG systems by Valverde et al. [11]. They found that high pass filters usually implemented in SAECG systems (with cutoff frequencies at $29 \mathrm{~Hz}$ and $40 \mathrm{~Hz}$ ) affects the $\mathrm{P}$-wave duration estimation in a manner that depended on the filters used.

In particular, they showed that each filter gave different values of sensitivity and sensitivity for the discrimination between paroxymal AF patients and a control group.

In this study, we investigated the effects of several high-pass filters applied to conventional ECG on the Pwave characteristics.

To do this, we used ECG acquired by a DC-coupled acquisition system, which do not provide any analog high-pass filter to the signal. The raw signals have been processed by 24 digital high pass filters.

We found that ECG filtering affects the estimation of $\mathrm{P}$-wave duration in a manner that depends upon the type of filter used. In particular we found that the unidirectional filter have the major effect on the P-wave duration estimation.

In conclusion, high pass filters used in conventional electrocardiographs should be taken into consideration when estimating $\mathrm{P}$-wave duration to discriminate patients at risk of developing AF.

Such a consideration acquire even greater importance when the P-wave morphological analysis is concerned. Indeed, in this case high-pass filters can affect the estimation of morphological parameters, which have been recently demonstrated to provide additional and crucial information about the atrial conduction in patients prone to $\mathrm{AF}[12]$.

The beat-to-beat linear piecewise interpolation implemented in this study and used as the reference signal, provoke only the TP and PQ track to become isoelectric (on the baseline).

Since this time-domain technique is more respective of the P-wave morphology, this technique could be suggested to remove baseline wonder when P-wave characteristics estimation is concerned.

\section{References}

[1] Dilaveris P, Gialafos J. Future concept in P-wave morphological analyses. Card Electrophysiol Rev 2002; 6:221-224

[2] Platonov PG, Carlson J, Ingemansson MP, Roijer A, Hansson A, Chireikin LV, Olsson SB. Detection of interatrial conduction defects with unfiltered signal-averaged $\mathrm{P}$ wave ECG in patients with lone atrial fibrillation. Europace 2000; 2:32-41.

[3] Kannel WB, Wolf PA, Benjamin EJ, Levy D. Prevalence, incidence, prognosis, and predisposing conditions for atrial fibrillation: population-based estimates. Am J Cardiol. 1998 Oct $16 ; 82(8 \mathrm{~A}): 2 \mathrm{~N}-9 \mathrm{~N}$.

[4] Dilaveris PE, Gialafos JE, Sideris S. Simple electrocardiographic markers for the prediction of paroxysmal idiopathic atrial fibrillation. Am Heart $\mathrm{J}$ 1998;135:73-8.

[5] Jordaens L, Tavernier R, Gorgov N, 'T Kindt H, Dimmer C, Clement D. Signal-averaged P-wave: predictor of atrial fibrillation. J cardiovasc Electrophysiol 1998; 9:S30-S34.

[6] Dilaveris PE, Gialafos JE. P-wave dispersion : a novel predictor of paroxysmal atrial fibrillation. A.N.E. 2001; 6:159-165.

[7] Darbar D, Jahangir A, Hammill SC, Gersh BJ. P-wave signal-averaged electrocardiography to identify the risk for atrial fibrillation. Pace 2002; 25:1447-1453.

[8] Michelucci A, Bagliani G, Colella A, Pieragnoli P, Porciani MC, Gensini G, Padeletti L. P-wave assessment: state of the art update. Card Electrophysiol Rev. 2002; 6:215-220

[9] Clavier L, Boucher JM, Lepage R, Blanc JJ, Cornily JC. Automatic P-wave analysis of patients prone to atrial fibrillation. Med Biol Eng Comput. 2002 Jan;40(1):63-71.

[10] Klein M, Evans SJ, Blumberg S, Cataldo L, Bodenheimer MM. Use of P-wave-triggered, P-wave signal-averaged electrocardiogram to predict atrial fibrillation after coronary artery bypass surgery. Am Heart J. 1995 May;129(5):895-901.

[11] Valverde ER, Quinteiro RA, Bertran GC, Arini PD, Glenny P, Biagetti MO. Influence of filtering techniques on the time-domain analysis of signal-averaged $\mathrm{P}$ wave electrocardiogram.J Cardiovasc Electrophysiol. 1998 Mar;9(3):253-60.

[12] Censi F, Calcagnini G, Ricci C, Ricci RP, Santini M, Grammatico A, Bartolini P. P-wave morphology assessment by a gaussian functions-based model in atrial fibrillation patients.IEEE Trans Biomed Eng. 2007 Apr;54(4):663-72.

Address for correspondence

Federica Censi

Dept. Technology and Health

Italian Institute of Health

Viale Regina Elena 299

00161 Roma

Italy

federica.censi@iss.it 